\title{
Metastasis from Prostatic Adenocarcinoma: A Mimicker of Chronic Subdural Hematoma
}

\section{Sreenath $\mathbf{K}^{1 *}$, Anand P Raghavan ${ }^{2}$, Bijukrishnan $\mathbf{R}^{3}$ and Arunkumar $\mathbf{R S}^{4}$}

${ }^{1}$ Senior Resident, Department of Neurosurgery, Government Medical College, Thrissur, Kerala

${ }^{2}$ Assistant Professor, Department of Neurosurgery, Government Medical College, Thrissur, Kerala

${ }^{3}$ Professor, Department of Neurosurgery, Government Medical College, Thrissur, Kerala

${ }^{4}$ Senior Resident, Department of Pathology, Government Medical College, Thrissur, Kerala

*Corresponding Author: Sreenath K, Senior Resident, Department of Neurosurgery, Government Medical College, Thrissur, Kerala.

Received: July 30, 2019; Published: October 30, 2019

DOI: 10.31080/ASNE.2019.02.0121

\begin{abstract}
Dural metastasis from non neurologic cancer is rare (8 to $9 \%$ of cases) and carcinoma prostate is the most common malignancy (19.5\% of cases) associated with carcinomatous involvement of dura. Here we report a case of prostatic dural metastasis which closely mimicked chronic subdural hematoma both clinically and radiologically and underwent initial treatment according to the guidelines for the treatment of chronic subdural hematoma. Patient is a known case of adenocarcinoma of prostate which was diagnosed 3 years back now presented with two days history of slurring of speech and weakness left side of the body. Patient also had history of fall. Non contrast CT brain showed iso-hypodense extra axial collection and suspected chronic subdural hematoma and underwent initial twist drill and later burr hole craniotomy. Intraoperatively dural en plaque lesion was noted and histopathology examination was suggestive of metastasis from prostatic adenocarcinoma. Hence authors conclude that dural metastais should be considered as a differential diagnosis in all patients of carcinoma prostate, if the patient comes with clinical or radiological features of chronic subdural hematoma.
\end{abstract}

Keywords: Adenocarcinoma; Chronic Subdural Hematoma; Metastasis

\section{Introduction}

A chronic subdural hematoma is a liquefied hematoma in the subdural space usually occurring at least 3 weeks after a head injury [1].

It is usually occurring in elderly patients and factors associated with increased risk of chronic subdural hematoma includes male sex, alcohol consumption, use of anticoagulant or antiplatelet drugs, Alzheimer's disease and other neurological diseases associated with brain atrophy, systemic disease associated with brain atrophy, such as liver and kidney disease, dialysis etc [1].

Carcinoma prostate rarely cause dural metastasis which may mimic a subdural hematoma both clinically and radiologically [2]. Here we report a case of prostatic dural metastasis which closely mimicked chronic subdural hematoma and underwent initial treatment according to the guidelines of chronic subdural hematoma.

\section{Case Report}

61 year old male presented with history of slurring of speech and weakness left side of body for 2 days duration. Patient also gave history of fall at home twice, 2 weeks and 2 month back. He was diabetic and was on insulin. He is a known case of adenocarcinoma of prostate which was diagnosed 3 years back and received chemotherapy for the same. No history of hypertension, cardiac illness, or other significant comorbidities. Patient was not an alcoholic, not on antiplatelets or no history of bleeding disorders. On examination patient was conscious, confused, GCS $14 / 15$, pupil equal in size and reacting normally to light, left hemiperesis, pulse rate 82 per minute, BP 110/70 mm Hg. All other blood investigations were within normal limits other than anemia ( $\mathrm{Hb} 9.1 \mathrm{~g} / \mathrm{dl})$. Non contrast CT brain showed iso-hypodense extra axial collection noted in right cerebral convexity with minimal effacement of adjacent sulcal spaces and ventricles suggestive of chronic subdural hematoma (Figure 1). Emergency twist drill craniostomy was done but no fluid came out. 
Follow up CT scan after the twist drill craniostomy showed persistence of chronic subdural hematoma even though the drain tip was inside hematoma (Figure 2). A Burr hole craniotomy was planned in view of persistence of subdural collection causing mass effect on the next day. Intra operatively it was observed that there was no subdural hematoma but instead of that there was dural thickening and en plaque lesion underneath. Total removal of the lesion was not done as we are not sure about the diagnosis. Biopsy was done and histopathology examination showed an infiltrating neoplasm composed of cells arranged in vague glandular pattern and fused glands and individual cells are having moderate amount of cytoplasm and round to oval hyperchromatic nucleus in a desmoplastic stroma suggestive of metastasis from adenocarinoma of prosate (Figure 3 and 4). Craniotomy and excision of lesion was advised but relatives were not willing for further surgical intervention after explaining the prognosis. Patient was referred to department of radiotherapy for further management. Patient went home at request and he expired 2 weeks later

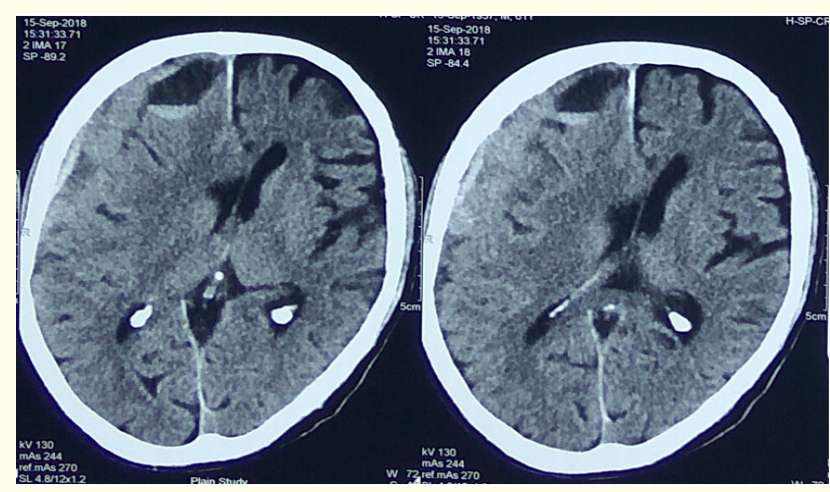

Figure 1: Pre operative NCCT brain.

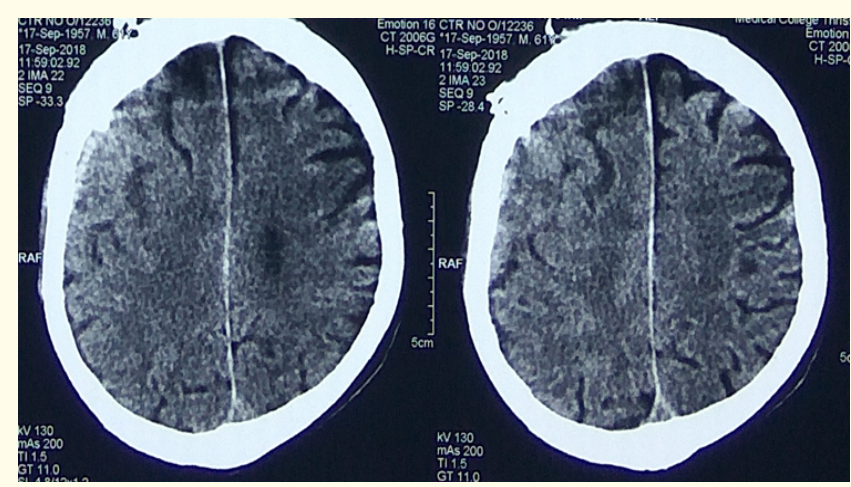

Figure 2: NCCT brain After twist drill craniostomy. Note The drain tip which is inside the lesion

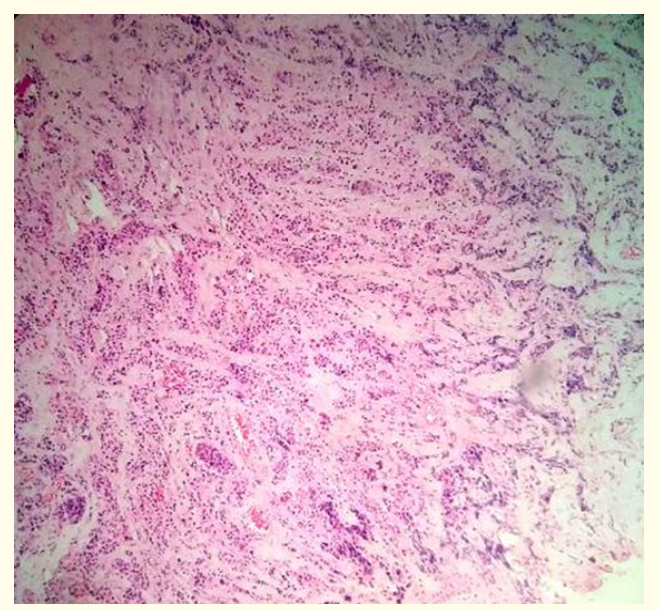

Figure 3: (Histopathology 10X magnification).

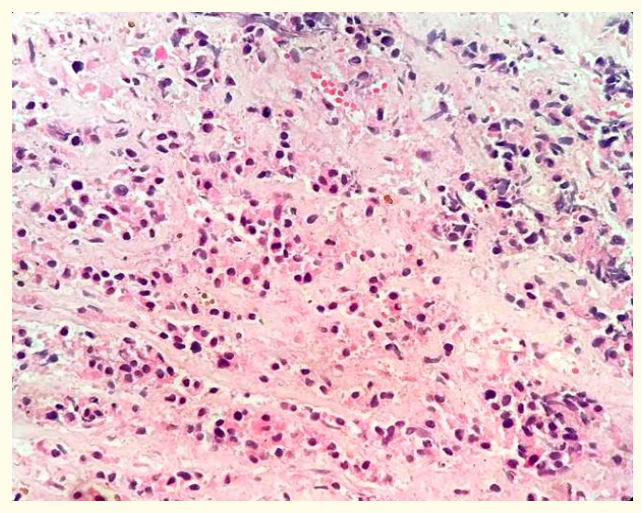

Figure 4: (Histopathology 40X magnification).

\section{Discussion}

A chronic subdural hematoma is a disease of elderly which may present with variety of symptoms such as headache, confusion, memory disturbances, motor deficits, seizures etc. It may commonly associated with past history of trauma and iso or hypo dense, homo or heterogenous in non contrast computed tomography of brain. 1 Dural metastasis can mimic chronic subdural hematoma both clinically and radiologically. According one study published in 2015 , there are only 11 cases reported as dural metastasis of prostate cancer mimicking a chronic subdural hematoma till that time [2].

Dural metastasis is caused by a variety of tumours such as carcinoma prostate, breast, lung and stomach. Dural metastases from carcinoma prostate occur due to the direct extension of skull metastasis or hematogenous spread or retrograde spread through the vertebral venous plexus $[3,4]$. Patients with dural metastasis from prostate cancer have worse prognosis and it indicates end stage 
of advanced disease [2]. One year survival rate of prostate cancer with brain metastasis is $18 \%$ and average survival is 7.6 months [5].

Brain CT scan may cause confusion in diagnosing and it can closely mimick chronic subdural hematoma. Hence contrast enhanced MRI brain is needed to confirm the presence of brain metastases [6]. Steroid therapy should be started early if there is significant mass effect due to tumour edema. Craniotomy and removal of the lesion may prolong the survival and radiotherapy may be indicated in these patients [7].

\section{Conclusion}

Carcinoma Prostate can cause dural metastasis which can closely mimic subhural hematoma. Hence dural metastais should be considered as a differential diagnosis in all patients of carcinoma prostate, if the patient comes with clinical or radiological features of chronic subdural hematoma.

\section{Bibliography}

1. Macdonald RL. "Pathophysiology of Chronic Subdural Hematomas". In: Winn R H. editor. Youmans and Winn Neurological Surgery.7th edition. Philadelphia: Elsevier (2017): 304-309.

2. Nzokou A., et al. "Subdural Metastasis of Prostate Cancer". Journal of Neurological Surgery Reports 76 (2015): 123-127.

3. Laigle-Donadey F., et al. "Dural metastases". Journal of NeuroOncology 75.1 (2005): 57-61.

4. Nayak L., et al. "Intracranial dural metastases". Cancer 115.9 (2009):1947-1953.

5. Fervenza FC., et al. "Brain metastasis: an unusual complication from prostatic adenocarcinoma". Mayo Clinic Proceedings 75 (2000): 79-82.

6. Merci Kabulo KD., et al. "Dural Metastasis with Subdural Hemorrhage from Prostate Cancer". International Journal of Neurologic Physical Therapy 3.6 (2017): 49-53.

7. Gupta A., et al. "Brain stem metastasis as the only site of spread in prostate carcinoma. A case report". Cancer 74 (1994): 25162519.

\section{Volume 2 Issue 11 November 2019}

(C) All rights are reserved by Sreenath K., et al. 